\title{
EXPLORANDO O POTENCIAL DE TAREFAS DE TEMPO DE REAÇÃO SIMPLES VISUAL PARA A EDUCAÇÃO FÍSICA: REVISÃO SISTEMÁTICA
}

\author{
Tânia Brusque Crocetta \\ Fábio Hech Dominski \\ Alexandro Andrade ${ }^{3}$
}

CROCETTA, T. B.; DOMINSKI, F. H.; ANDRADE, A. Explorando o potencial de tarefas de tempo de reação simples visual para a educação física: revisão sistemática. Arq. Cienc. Saúde UNIPAR, Umuarama, v. 18, n. 2, p, 127-140, maio/ago. 2014.

\begin{abstract}
RESUMO: Este estudo teve como objetivo rever criticamente a literatura científica publicada na última década (2003 a 2012) sobre o uso de testes de tempo de reação simples com estímulo visual relacionada à Educação Física, mais especificamente objetiva apresentar uma síntese da evidência empírica disponível sobre a eficácia do uso deste tipo de teste no esporte, exercício e atividade física, buscando na base de dados eletrônica Thomson Reuters Web of Knowledge $\mathrm{SM}^{\mathrm{SM}}$. A partir das combinações de palavras "simple reaction time e athlete", "simple reaction time e sport", "simple reaction time e exercise" e "simple reaction time e physical activity", 36 artigos foram selecionados para o estudo. As categorias investigadas foram: periódico, número de participantes, sexo e idade, população investigada, software utilizado, tema e resultados. As publicações se concentraram nas revistas Medicine and science in sports and exercise e Psychology of sport and exercise, ambas com $n=3$. Predominaram pesquisas com o uso de testes de tempo de reação simples com estímulo visual (TRSimples) para avaliação do efeito do exercício físico sobre o desempenho no TRSimples ( $\mathrm{n}=13$ ) com uma média de 93 participantes (11 a 551 sujeitos). As populações de atletas investigadas foram variadas, com predominância do futebol e atletismo (ambas com n=5). A maioria dos estudos utilizou o software ANAM (Automated Neuropsychological Assessment Metrics) como instrumento de coleta de dados do TRSimples. Os artigos foram identificados e apresentados quanto ao seu tema de pesquisa: a) Uso do TRSimples para avaliação da concussão cerebral causado por traumatismo na cabeça $(n=8)$; b) Efeito do exercício físico sobre o desempenho no TRSimples $(n=13)$; c) Níveis de prática e comparações do TRSimples de atletas e não atletas $(n=8)$ e d) Fatores que influenciam o TRSimples ( $n=7)$. Os resultados dos artigos apontaram associações positivas entre o uso de testes de TRSimples e a concussão cerebral originada pelo esporte além do efeito positivo do exercício físico sobre o desempenho cognitivo. O TRSimples contribuiu para análise dos níveis de prática de atletas de mesma modalidade, de modalidades distintas e de não atletas, além de ser um indicador sensível a diversos fatores que podem influenciar o desempenho cognitivo.
\end{abstract}

PALAVRAS-CHAVE: Tempo de reação; Software; Esporte; Exercício; Atividade física.

\section{SIMPLE REACTION TIME SOFTWARE AND ITS APPLICATIONS IN SPORT, EXERCISE AND PHYSICAL ACTIVITY: A SYSTEMATIC REVIEW}

\begin{abstract}
This study aimed to review the literature published in the last decade (2003 to 2012) about the use of simple reaction time tests with visual stimuli (TRSimple) presenting a synthesis of the empirical evidence available about the effectiveness of using this type of testing in sport, exercise and physical activity. From the combinations of the words "simple reaction time" and "athlete", "sport", "exercise" or "physical activity", 36 articles were selected for the study. The categories investigated were journal, number of participants, age and sex, population, software used, theme and results. The publications focused on the journals Medicine and science in sports and exercise and Psychology of sport and exercise, both with $\mathrm{n}=3$. There was a predominance of research using simple reaction time tests with visual stimuli for evaluating the effect of exercise over performance on TRSimple ( $\mathrm{n}=13$ ) with an average of 93 participants (11 to 551$)$. The populations of athletes investigated varied, with a predominance of soccer players and athletics (both with $\mathrm{n}=5$ ). Most studies used the ANAM (Automated Neuropsychological Assessment Metrics) software as a tool for collecting TRSimple. The articles were identified and presented regarding their research topic: a) Use of TRSimple to evaluate concussion caused by head trauma ( $n=8)$; b) Effects of exercise on performance in TRSimple ( $n=13)$; c) Levels of practice and TRSimple comparisons of athletes and non-athletes $(n=8)$ e d) Factors that influence the TRSimple ( $\mathrm{n}=7)$. The results of the articles showed positive associations in the use of TRSimple tests and concussion caused by sport beyond the positive effect of exercise on cognitive performance. TRSimple contributed to the analysis of the practice levels of athletes in the same sport, in different modalities and non-athletes, besides being a sensitive indicator to several factors that may influence cognitive performance.
\end{abstract}

KEYWORDS: Reaction time; Software; Sport; Exercise; Physical activity.

\section{Introdução}

O grau de sucesso ou fracasso experimentado no desempenho de uma tarefa específica é muitas vezes, determinado pela velocidade inicial na qual se reage a determinado estímulo e esta variabilidade da velocidade de resposta neuromuscular humana tem sido um problema de considerável preocupação manifestada por psicólogos e educadores físicos (O’BRIEN, 1959).
Estes testes que medem o tempo necessário para um sujeito responder à apresentação de estímulos são convencionalmente denominados "Testes de Tempo de Reação" e o tempo de reação total para a resposta a estes testes consiste de duas partes: a) o Tempo de Reação (TR), que é o tempo necessário para reconhecer o estímulo e b) o Tempo de Movimento (TM), que é o tempo a partir do reconhecimento do estímulo até a indicação da resposta (KELLY; MURPHY; BACKHOUSE, 2000; LOUREIRO; DE FREITAS, 2012).

DOI: https://doi.org/10.25110/arqsaude.v18i2.2014.5178

${ }^{1}$ Mestre em Ciências do Movimento Humano pelo Centro de Ciências da Saúde e do Esporte - CEFID da Universidade do Estado de Santa Catarina UDESC, pesquisadora associada ao Laboratório de Psicologia do Esporte e do Exercício - LAPE. Rua Pascoal Simone, 358 - Coqueiros, Florianópolis - SC, CEP 88080-350, Fone (48) 33218677, tania.crocetta@udesc.br

${ }^{2}$ Graduando em Bacharelado em Educação Física pelo CEFID/UDESC, bolsista pesquisador do LAPE. Rua Pascoal Simone, 358 - Coqueiros, Florianópolis - SC, CEP 88080-350, Fone (48) 33218677, fabiohdominski@hotmail.com

${ }^{3}$ Prof. Doutor do Programa de Pós-graduação Strictu Sensu em Ciências do Movimento Humano do CEFID/UDESC, coordenador do LAPE. Rua Pascoal Simone, 358 - Coqueiros, Florianópolis - SC, CEP 88080-350, Fone (48) 33218677, alexandro.andrade@udesc.br 
O TR refere-se ao tempo desde o início do estímulo com o início de uma evidente resposta, em que na maioria dos estudos, esta resposta é o pressionamento ou liberação de um botão (DRAPER; MCMORRIS; PARKER, 2010). Assim, uma vez que exista algum movimento, mesmo que pequeno, considera-se como um teste de Tempo de Reação Total (TRT).

O tempo de reação é o tempo decorrido entre a apresentação de um estímulo sensorial e a resposta comportamental posterior. TR é mais rápido quando há apenas uma resposta possível (tempo de reação simples) e, torna-se mais lento quando opções de resposta são adicionadas (TR escolha) (CHANDRA et al., 2010).

É consenso entre os pesquisadores que existem testes distintos de TR que podem ser simples, obtidos em testes onde os sujeitos respondem a estímulos como luz ou som; de reconhecimento, que são testes com dois tipos de estímulos em que um deve ser para resposta do sujeito e o outro serve como uma distração que deve ser ignorada (também conhecido como teste de GoNoGo); e escolha, quando o sujeito precisa selecionar uma resposta a partir de um conjunto de respostas possíveis (BAAYEN, 2010). O aumento no tempo de desempenho de um teste de TR mais complexo se deve ao fato deste requerer funções cognitivas adicionais quando comparado a um teste de TR simples, porém, o TM deve ser similar nos dois testes (KELLY; MURPHY; BACKHOUSE, 2000).

Neste estudo utilizou-se o conceito de Ishihara, Imanaka e Mori, (2002) e Ellemberg, St-Louis-Deschenes, (2010), em que o Tempo de Reação Total (TRT) é descrito como o tempo total necessário para o processamento de informações em três fases: identificação de estímulo (sensorial ou perceptiva), a seleção da resposta (decisão ou cognitiva) e a programação da resposta (motora).

O TRT é uma medida simples que contribui para o entendimento do desempenho cognitivo de pessoas que são fisicamente ativas, em praticantes de esporte ou mesmo fisiologistas e treinadores, uma vez que em várias atividades esportivas ou atividades de recreação a percepção periférica tem um papel fundamental na prática adequada e segura (JIMÉNEZ-PAVÓN et al., 2011).

A identificação da diferença em cada componente do TRT permite o entendimento dos efeitos do treinamento esportivo, do exercício e da atividade física na transmissão neural e o acoplamento da excitação-contração no controle motor (CHUNG; NG, 2012), determinar qual o componente do TRT que é responsável pelo atraso na avaliação pode fornecer informações úteis sobre os processos de programação no sistema nervoso central (GUNENDI; TASKIRAN; BEYAZOVA, 2005) ou ainda, um modelo que faça uma distinção entre as funções executivas e outros processos cognitivos pode começar a distinguir as razões pelas quais algumas funções cognitivas são reforçadas pelo exercício, enquanto outras são prejudicadas (DIETRICH; AUDIFFREN, 2011).

Em uma revisão sistemática conduzida por Mcdonnell, Smith e Mackintosh (2011) para avaliar se o exercício aeróbio melhora a cognição em adultos diagnosticados com distúrbios neurológicos, as autoras concluíram que os resultados de estudos específicos mostram que o exercício aeróbio melhora a cognição em pessoas com demência, melhora a atenção e flexibilidade em pacientes com lesão cerebral trau- mática, melhora o TRT de escolha em pessoas com esclerose múltipla, e melhora o aprendizado motor em pessoas com acidente vascular cerebral crônico.

Muitos testes tradicionais aplicados em lápis e papel não foram projetados para detectar pequenas reduções nas respostas cognitivas, enquanto que discriminações de tais mudanças, além da capacidade de medição de testes neuropsicológicos clínicos tradicionais, são facilmente detectados usando protocolos de avaliação informatizados (ERLANGER et al., 2003).

Estudos propõem testes de TRT com o uso de softwares executados em computador com seus acessórios para registro da resposta, mostrando-se eficazes na verificação de que o controle cognitivo de esgrimistas é mais beneficiado a partir da combinação de treinamento físico e mental quando comparado à administração de cada um isoladamente (CHAN et al., 2011); que o TRT de atletas de tae-kwon-do é menor para aqueles com maior tempo de prática (ANDRADE; BELMONTE; VIANA, 2006); já o estudo de Andrade et al. (2005) demonstrou que o tempo de reação não é um fator preditor de performance em atletas de tênis de campo, sendo que, o que pode diferenciar um jogador mais experiente de um iniciante pode ser o tempo de processamento da informação, e não o tempo para a reação, especificamente.

Um teste de TRT simples em que os sujeitos movem uma articulação tão rapidamente quanto possível, em resposta a uma indicação visual pode ser utilizado para avaliar a capacidade motora, bem como a capacidades de tomada de decisão em atletas (YOTANI et al. 2011). Porém, apesar da importância do tempo de movimento no esporte, pouca pesquisa foi realizada para examinar o efeito do exercício sobre este aspecto do TRT (DRAPER; MCMORRIS; PARKER, 2010).

A literatura nos mostra que a Psicologia do Esporte e do Exercício vem se apropriando de testes de TR em seus estudos. Kornspan (2007) afirma que um dos primeiros estudos a investigar os aspectos psicológicos do esporte utilizou um teste de TR em esgrimistas (KORNSPAN, 2007). Como as atividades esportivas podem ser estudadas de diferentes maneiras e em diferentes níveis (BOSCHKER; BAKKER; MICHAELS, 2002), a discussão em torno da produção científica sobre o uso de testes de tempo de reação em atletas, em praticantes de exercício ou atividade física pode beneficiar o desenvolvimento do esporte e da atividade física, em especial apoiando ações para aumento da qualidade de vida, como também da Psicologia do Esporte e do Exercício enquanto área científica.

Os testes de tempo de reação simples com estímulo visual (TRSimples) estão sendo usados no âmbito da Educação Física e da Psicologia do Esporte e do Exercício para avaliar o efeito do exercício e da atividade física para melhorar a saúde e estado físico de diversas populações. Essa afirmação precisa ser reforçada com as experiências de aplicação e, acima de tudo, pela evidência empírica.

Visando contribuir com a sistematização do conhecimento produzido na área, o objetivo deste estudo foi analisar a produção científica relacionada ao uso de testes de TRsimples com estímulo visual relacionado à Educação Física para apresentar uma síntese da evidência empírica disponível sobre a eficácia do uso deste tipo de teste em atletas, praticantes de exercício ou atividade física. 


\section{Material e Método}

Trata-se de uma revisão sistemática da literatura, na qual os dados utilizados para a investigação são provenientes de estudos selecionados por meio de um método sistemático (ANDRADE et al., 2012) descrito a seguir.

\section{Estratégia de Busca}

Este estudo delimitou-se a investigar a produção científica sobre o uso de testes de tempo de reação simples com estímulo visual (TRSimples) em atletas, praticantes de exercício ou atividade física publicada em periódicos indexados na base de dados eletrônica Thomson Reuters Web of Knowledge $^{\mathrm{SM}}$. A busca pelos artigos foi realizada no mês de janeiro de 2013.

A Thomson Reuters Web of Knowledge ${ }^{\mathrm{SM}}$ atende aos critérios de abrangência e representatividade, por se tratar de uma plataforma de pesquisa que dá acesso ao conteúdo objetivo com cobertura de mais de 256 disciplinas na área das ciências, ciências sociais, artes e humanidades, disponibilizando as plataformas Web of Science ${ }^{\mathrm{SM}}$ (com mais de 12.000 revistas de alto impacto e mais de 148 mil artigos de conferências), Chinese Science Citation Database (base de dados composta por quase 1.100 das principais publicações acadêmicas da China, com quase dois milhões de registros), Current Contents Connect ${ }^{\circledR}$ (cerca de 8.000 jornais e 2.000 livros), Medline $\AA$, Derwent Innovations Index ${ }^{S M}$, BIOSIS Citation Index ${ }^{S M}$, BIOSIS Previews $\AA$, Biological Abstracts ${ }^{\circledR}$, CABI: CAB Abstracts ${ }^{\circledR}$, Global Health ${ }^{\circledR}$, Inspec ${ }^{\circledR}$, Food Science and Technology, Abstracts ${ }^{\mathrm{TM}}$, Zoological Record ${ }^{\circledR}$, Journal Citation Reports ${ }^{\circledR}$ e Essential Science Indicators ${ }^{\circledR}$ (REUTERS, 2013).

A partir das combinações de palavras "simple reaction time e athlete", "simple reaction time e sport", "simple reaction time e exercise" e "simple reaction time e physical activity" 36 artigos foram selecionados para a pesquisa (Figura 1). Os itens investigados foram: referência, periódico, número de participantes, sexo e idade, população investiga$\mathrm{da}$, instrumento utilizado (software), tema (categorizados em A, B, C ou D) e resultados.

Foram utilizados como filtro para a pesquisa os termos encontrados no resumo, título e palavras-chave. Como abrangência da busca, delimitaram-se os artigos publicados no período compreendido entre os anos de 2003 e 2012, contemplando os últimos 10 anos.
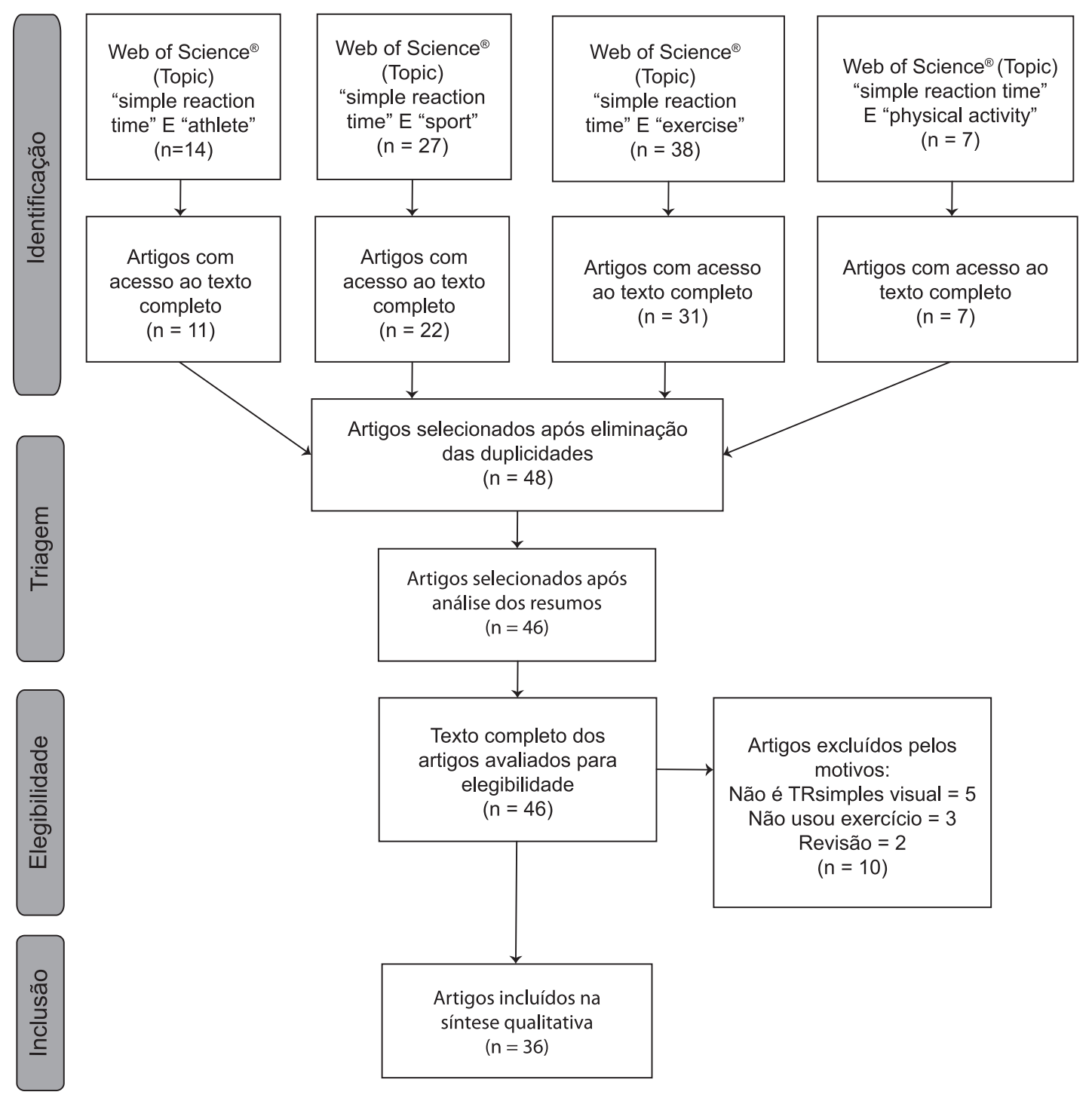

Figura 1: Processo de seleção de estudos relacionados ao tempo de reação em atletas, em praticantes de exercício e atividade física publicados na base de dados Thomson Reuters Web of Knowledge ${ }^{\mathrm{SM}}$ nos anos de 2003 a 2012. Fonte: adaptado de Moher et al., (2009) 
Após seleção dos artigos $(\mathrm{n}=36)$, todos foram lidos na íntegra por dois pesquisadores. Após a leitura, os artigos foram tabulados mediante características identificadas como relevantes para a presente revisão sistemática, considerando os seguintes itens: a) referência, b) periódico, c) número de participantes, d) amostra (sexo e idade), e) população investigada, f) instrumento utilizado, g) tema e h) resultado.

A análise dos artigos selecionados por meio das categorias produziu resultados analisados e discutidos segundo os seguintes temas: a) Uso do TRSimples para avaliação da concussão cerebral; b) Efeito do exercício físico sobre o desempenho no TRSimples; c) Níveis de prática e comparações do TRSimples de atletas e não atletas; e d) Fatores que influenciam o TRSimples.

\section{Critérios de inclusão}

Foram incluídos artigos com texto completo, que utilizaram um teste de TRSimples que analisou a influência do exercício ou da atividade física no TR, ou que comparou ou correlacionou o TR com outras características da amostra utilizada.

\section{Resultados}

Foram encontrados 86 artigos na base de dados pesquisada e após a exclusão dos duplicados e daqueles que não se enquadraram no tema, por meio da leitura do título e do resumo, restaram 36 artigos que compuseram a amostra analisada. As razões mais comuns para exclusão de artigos foram aqueles que não utilizaram um teste de tempo de reação simples com estímulo visual (TRSimples), que não envolviam amostra de atletas ou qualquer relação com o esporte, exercício ou atividade física. Uma revisão não incluída na seleção (DIETRICH; AUDIFFREN, 2011) foi utilizada para compor a discussão.

As características dos artigos incluídos neste estudo estão descritas nas Tabelas 1, 2 e 3 com as seguintes categorias: referência, periódico no qual foi publicado, número de participantes, amostra (sexo e idade), população investigada, instrumento utilizado para medir o TRSimples, tema de pesquisa (agrupados e identificados pelas letras a, b, c e d para posterior discussão) e principais resultados.

Dos 36 artigos analisados, as publicações se concentraram em duas revistas, o Medicine and Science in Sports and Exercise e a Psychology of Sport and Exercise, ambas com 3 publicações. Em relação ao número de participantes os artigos variaram entre 10 a 3.802 sujeitos (média de 260).

Tabela 1: Características dos estudos incluídos para a seleção por "Tempo de reação simples" e "Athlete" (ordenados alfabeticamente)

\begin{tabular}{|c|c|c|c|c|c|c|c|}
\hline Referência & & $\begin{array}{c}\mathrm{N} \\
\text { Sexo } \\
\text { Idade }\end{array}$ & População & Instrumento & Tema & Cat & Resultado \\
\hline
\end{tabular}

\begin{tabular}{|c|c|c|c|c|c|}
\hline $\begin{array}{c}\text { (BAILEY et } \\
\text { al., 2010) }\end{array}$ & 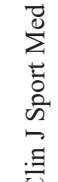 & $\begin{array}{c}47 \\
\mathrm{M} \\
17-49\end{array}$ & $\begin{array}{l}\text { Jogadores de } \\
\text { futebol Ame- } \\
\text { ricano. }\end{array}$ & CRI & $\begin{array}{l}\text { Avaliar o efeito do estresse psi- } \\
\text { cológico sobre o desempenho } \\
\text { neurocognitivo após pancada na } \\
\text { cabeça. }\end{array}$ \\
\hline
\end{tabular}

Correlações significativas entre os aspectos somáticos como esquizofrenia, preocupação, depressão, ansiedade,

A abuso de drogas e ideias suicidas e o cologico sobre o desempenho A $\begin{aligned} & \text { abuso de drogas e ideias suicidas e } \\ & \text { neurocognitivo após pancada na }\end{aligned}$ -
desenho no TR, e quando examinados categoricamente, os participantes com ideias suicidas apresentaram um TR mais lento.

\begin{tabular}{|c|c|c|c|c|c|c|c|}
\hline $\begin{array}{l}\text { (BROWN; } \\
\text { GUSKIEWI- } \\
\text { CZ; BLEI- } \\
\text { BERG, 2007) }\end{array}$ & 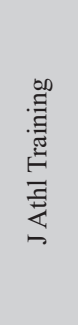 & $\begin{array}{l}327 \\
\mathrm{Me} \mathrm{F} \\
18^{*}\end{array}$ & $\begin{array}{l}\text { Futebol, } \\
\text { lacrosse, bas- } \\
\text { quete, hóquei } \\
\text { de campo, } \\
\text { futebol ame- } \\
\text { ricano, luta, } \\
\text { salto em altu- } \\
\text { ra, salto com } \\
\text { vara e torcida } \\
\text { organizada. }\end{array}$ & ANAM & $\begin{array}{l}\text { Avaliar o desempenho neuro- } \\
\text { cognitivo e os efeitos de fatores } \\
\text { como sexo, estado de alerta e } \\
\text { tipo de esporte. }\end{array}$ & D & $\begin{array}{l}\text { Desempenho pode ser afetado por uma } \\
\text { série de fatores, incluindo sexo, estado } \\
\text { de alerta no momento do teste e pelo } \\
\text { esporte praticado pelo atleta. }\end{array}$ \\
\hline $\begin{array}{l}\text { (CHADDO- } \\
\text { CK et al., } \\
\text { 2011) }\end{array}$ & 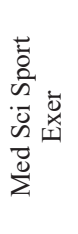 & $\begin{array}{l}36 \\
\mathrm{M} \text { e F } \\
19-24\end{array}$ & $\begin{array}{l}\text { Basquete, } \\
\text { corredor, gi- } \\
\text { nasta, futebol, } \\
\text { natação, tênis, } \\
\text { atleta de pista } \\
\text { e campo, luta } \\
\text { e não atletas. }\end{array}$ & E-Prime software & $\begin{array}{l}\text { Verificar se atletas mostram de- } \\
\text { sempenho superior na tarefa de } \\
\text { atravessar a rua e que isto está } \\
\text { relacionado com a velocidade de } \\
\text { processamento mais rápido na } \\
\text { tarefa de TR simples. }\end{array}$ & $\mathrm{C}$ & $\begin{array}{l}\text { Dados sugerem que as habilidades } \\
\text { cognitivas treinadas no esporte podem } \\
\text { ser transferidas para o desempenho de } \\
\text { tarefas do cotidiano. }\end{array}$ \\
\hline $\begin{array}{l}\text { (COJOCA- } \\
\text { RIU, 2011) }\end{array}$ & $\frac{\overrightarrow{0}}{\frac{\hbar}{0}}$ & $\begin{array}{c}73 \\
\mathrm{M} \\
18-33\end{array}$ & $\begin{array}{l}\text { Atletas de } \\
\text { Qwan Ki Do e } \\
\text { não atletas. }\end{array}$ & Próprio & $\begin{array}{l}\text { Identificar o tempo de reação } \\
\text { simples e de escolha em prati- } \\
\text { cantes iniciantes e avançados do } \\
\text { Qwan Ki Do. }\end{array}$ & $\mathrm{C}$ & $\begin{array}{l}\text { Não houve diferença significativa en- } \\
\text { tre o TRsimples de atletas e não atletas. } \\
\text { Recomenda que os treinadores testem } \\
\text { o TRsimples e de escolha para a sele- } \\
\text { ção de atletas de alta performance. }\end{array}$ \\
\hline $\begin{array}{l}\text { (ECKNER; } \\
\text { KUTCHER; } \\
\text { RICHARD- } \\
\text { SON, 2011) }\end{array}$ & 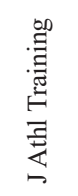 & $\begin{array}{l}102 \\
\mathrm{M} \text { e F } \\
>=18\end{array}$ & $\begin{array}{l}\text { Futebol ame- } \\
\text { ricano, futebol } \\
\text { e luta. }\end{array}$ & $\begin{array}{l}\text { CogState Sport } \\
\text { e régua adaptada } \\
\text { pelo autor }\end{array}$ & $\begin{array}{l}\text { Avaliar a confiabilidade de um } \\
\text { instrumento clínico (uma régua) } \\
\text { com um software computadori- } \\
\text { zado para análise do desempenho } \\
\text { motor após pancada na cabeça. }\end{array}$ & A & $\begin{array}{l}\text { O instrumento clínico proposto assim } \\
\text { como o comprometimento do desem- } \\
\text { penho no TR é apenas um dos mui- } \\
\text { tos sinais típicos que indicam que um } \\
\text { atleta tenha sofrido uma contusão na } \\
\text { cabeça. }\end{array}$ \\
\hline
\end{tabular}




\begin{tabular}{|c|c|c|c|c|c|c|c|}
\hline $\begin{array}{l}\text { (ERLAN- } \\
\text { GER et al., } \\
\text { 2003) }\end{array}$ & 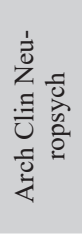 & $\begin{array}{l}823 \\
\mathrm{M} \mathrm{e} \mathrm{F} \\
13-35\end{array}$ & Adultos. & $\begin{array}{l}\text { CRI, Wechsler } \\
\text { Adult Intelligence } \\
\text { Scale, SDMT, } \\
\text { TMT, Lafayette } \\
\text { Grooved Pegbo- } \\
\text { ard Test, STP }\end{array}$ & $\begin{array}{l}\text { Identificar mudanças cognitivas } \\
\text { ocorridas por lesão após pancada } \\
\text { na cabeça. }\end{array}$ & A & $\begin{array}{l}\text { A bateria de testes do Concussion } \\
\text { Resolution Index (CRI) é um méto- } \\
\text { do válido e confiável para determinar } \\
\text { mudanças na velocidade psicomotora } \\
\text { e na velocidade de processamento de } \\
\text { informações para acompanhar lesões } \\
\text { na cabeça relacionada ao esporte. }\end{array}$ \\
\hline $\begin{array}{l}\text { (LOUREI- } \\
\text { RO; DE } \\
\text { FREITAS, } \\
\text { 2012) }\end{array}$ & 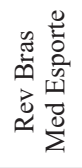 & $\begin{array}{c}24 \\
\mathrm{M} \\
18-32\end{array}$ & $\begin{array}{l}\text { Atletas de ba- } \\
\text { dminton. }\end{array}$ & LabView & $\begin{array}{l}\text { Investigar se jogadores de bad- } \\
\text { minton de alto nível apresentam } \\
\text { melhores índices de desempenho } \\
\text { neuromuscular do que jogadores } \\
\text { de nível intermediário. }\end{array}$ & $\mathrm{C}$ & $\begin{array}{l}\text { Jogadores de badminton de alto nível } \\
\text { reagem mais rapidamente a um estí- } \\
\text { mulo visual do que jogadores de nível } \\
\text { inferior durante uma tarefa de apontar } \\
\text { um alvo. }\end{array}$ \\
\hline $\begin{array}{l}\text { (LUFT; } \\
\text { TAKAS; } \\
\text { DARBY, } \\
\text { 2009) }\end{array}$ & 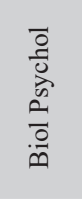 & $\begin{array}{l}30 \\
\mathrm{M} \mathrm{e} \mathrm{F} \\
16-25\end{array}$ & $\begin{array}{l}\text { Atletas de al- } \\
\text { to-rendimento } \\
\text { no atletismo. }\end{array}$ & CogState & $\begin{array}{l}\text { Variabilidade da frequência car- } \\
\text { díaca e desempenho cognitivo } \\
\text { antes e após o esforço físico. }\end{array}$ & B & $\begin{array}{l}\text { Resultados indicam que tempo de re- } \\
\text { ação mais rápido está relacionado à } \\
\text { menor variabilidade da frequência car- } \\
\text { díaca, o que sugere que a modulação } \\
\text { simpática superior está relacionada } \\
\text { com respostas rápidas. }\end{array}$ \\
\hline $\begin{array}{l}\text { (REGISTER- } \\
\text {-MIHALIK } \\
\text { et al., 2007) }\end{array}$ & 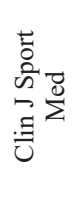 & $\begin{array}{l}247 \\
\mathrm{M} \mathrm{e} \mathrm{F} \\
16^{*}\end{array}$ & $\begin{array}{l}\text { Atletas de } \\
\text { basquete, } \\
\text { futebol ameri- } \\
\text { cano, futebol } \\
\text { ou lacrosse. }\end{array}$ & ANAM & $\begin{array}{l}\text { Examinar os efeitos das dores de } \\
\text { cabeça causadas por concussão, } \\
\text { na função neurocognitiva. }\end{array}$ & A & $\begin{array}{l}\text { Resultados demonstram aumento nos } \\
\text { déficits neurocognitivos após con- } \\
\text { cussão e a dor de cabeça pode ser um } \\
\text { indicador de diminuição da função } \\
\text { neurológica e / ou pode ser um fator } \\
\text { causador do aumento dos déficits. }\end{array}$ \\
\hline $\begin{array}{l}\text { (SCHMIDT } \\
\text { et al., 2012) }\end{array}$ & 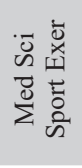 & $\begin{array}{l}1060 \\
\mathrm{M} \mathrm{e} \mathrm{F} \\
18 *\end{array}$ & $\begin{array}{l}\text { Atletas do } \\
\text { colegial. }\end{array}$ & ANAM & $\begin{array}{l}\text { Se existe acordo na identificação } \\
\text { de deficiências neurocognitivas } \\
\text { imediatas após contusão na ca- } \\
\text { beça usando dados individuais e } \\
\text { média por gênero. }\end{array}$ & A & $\begin{array}{l}\text { Resultados indicam que, para a maioria } \\
\text { dos resultados pós concussão, métodos } \\
\text { de comparação individual e média por } \\
\text { gênero identificam as mesmas defici- } \\
\text { ências. }\end{array}$ \\
\hline $\begin{array}{l}\text { (YOTANI et } \\
\text { al., 2011) }\end{array}$ & 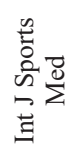 & $\begin{array}{c}14 \\
\mathrm{M} \\
20-22\end{array}$ & $\begin{array}{l}\text { Beisebol, vo- } \\
\text { leibol, Kendo, } \\
\text { atletismo, } \\
\text { futebol ou } \\
\text { golfe. }\end{array}$ & Não mencionou & $\begin{array}{l}\text { Determinar se o treinamento re- } \\
\text { duz o tempo visuo-motor de atle- } \\
\text { tas na execução de uma tarefa de } \\
\text { tempo de reação simples. }\end{array}$ & $\mathrm{C}$ & $\begin{array}{l}\text { Indivíduos que tiveram um maior } \\
\text { tempo antes do treino mostraram uma } \\
\text { maior redução no tempo após o treina- } \\
\text { mento. }\end{array}$ \\
\hline
\end{tabular}

$\overline{\mathrm{M}=\text { masculino; } \mathrm{F}=\text { feminino; }}{ }^{*}=$ média de idade dos participantes; ANAM=Automated Neuropsychological Assessment Metrics; CRI=Concussion Resolution Index; Id=Identificação do agrupamento dos temas da discussão, sendo: a) Uso do TRSimples para avaliação da concussão cerebral; b) Efeito do exercício físico sobre o desempenho no TRSimples; c) Níveis de prática e comparações do TRSimples de atletas e não atletas; e d) Fatores que influenciam o TRSimples.

Tabela 2: Características dos estudos incluídos para a seleção por "Tempo de reação simples" e "Sport" (ordenados alfabeticamente)

\begin{tabular}{|c|c|c|c|c|c|c|c|}
\hline Referência & : & $\begin{array}{c}\mathbf{N} \\
\text { Sexo } \\
\text { Idade }\end{array}$ & População & Instrumento & Tema & Cat & Resultado \\
\hline
\end{tabular}

\begin{tabular}{|c|c|c|c|c|c|}
\hline $\begin{array}{l}\text { (BAUR et } \\
\text { al., 2006) }\end{array}$ & 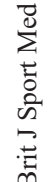 & $\begin{array}{c}18 \\
\mathrm{M} \\
26^{*}\end{array}$ & $\begin{array}{l}\text { Pilotos de corrida e } \\
\text { praticantes de corri- } \\
\text { da, natação, ciclismo, } \\
\text { basquetebol ou } \\
\text { futebol. }\end{array}$ & $\begin{array}{l}\text { Vienna Test } \\
\text { System }\end{array}$ & $\begin{array}{l}\text { Analisar a adequação de pi- } \\
\text { lotos de elite com relação ao } \\
\text { tempo de reação, a capacida- } \\
\text { de de desempenho da estabi- } \\
\text { lidade e de força. }\end{array}$ \\
\hline
\end{tabular}

\begin{tabular}{|c|c|c|c|c|}
\hline $\begin{array}{l}\text { (CHAN et } \\
\text { al., 2011) }\end{array}$ & 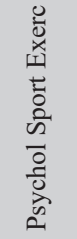 & $\begin{array}{c}60 \\
\mathrm{M} \text { e F } \\
18-26\end{array}$ & $\begin{array}{c}\text { Esgrimistas e univer- } \\
\text { sitários. }\end{array}$ & $\begin{array}{c}\text { Não mencio- } \\
\text { nou }\end{array}$ \\
\hline $\begin{array}{l}\text { (CHAN- } \\
\text { DRA et al., } \\
2010)\end{array}$ & 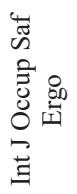 & $\begin{array}{c}15 \\
\mathrm{M} \\
24^{*}\end{array}$ & Pós-graduandos. & $\begin{array}{c}\text { Não usou } \\
\text { computador } \\
\text { (instrumento } \\
\text { elétrico para } \\
\text { avaliação do } \\
\text { TR) }\end{array}$ \\
\hline
\end{tabular}

TR)
Investigar os efeitos da experiência de esgrima e de aptidão física no controle inibitório de esgrimistas e não esgrimistas.
Pilotos apresentam tempos de reação mais rápidos do que fisicamente ativos praticantes de outras modalidades esC portivas. De acordo com os requisitos de corridas de automóveis, a força e a capacidade de desempenho sensório podem ser potencialmente melhorados.

Experiência de esgrima e de aptidão física facilitam a capacidade de reter uma ação quando necessário. Resultados sugerem que os benefícios do controle cognitivo é maior na combinação de treinamento físico e mental, do que quando comparado a cada um isoladamente.

Resultados indicaram uma diminuição Avaliar o efeito do exercício físico moderado e carga de calor graduada com o exercício em tarefas de tempo de reação. dos tempos de reação após o exercício,

D mas um aumento acentuado quando o exercício foi realizada a temperaturas elevadas. 


\begin{tabular}{|c|c|c|c|c|c|c|c|}
\hline $\begin{array}{l}\text { (DANE; } \\
\text { HAZAR; } \\
\text { TAN, 2008) }\end{array}$ & 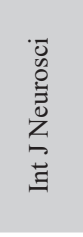 & $\begin{array}{l}88 \\
\mathrm{M} \mathrm{e} \mathrm{F} \\
12-14\end{array}$ & $\begin{array}{l}\text { Jogadores de Bad- } \\
\text { minton. }\end{array}$ & $\begin{array}{l}\text { Não usou } \\
\text { computador } \\
\text { (Newtest } \\
\text { 2000 Reac- } \\
\text { tion Time } \\
\text { Apparatus) }\end{array}$ & $\begin{array}{l}\text { Examinar as relações de } \\
\text { tempos de reação simples } \\
\text { das mãos direita e esquerda, } \\
\text { com força muscular da mão } \\
\text { direita e esquerda, costas e } \\
\text { pernas. }\end{array}$ & B & $\begin{array}{l}\text { Pode-se afirmar que o exercício pode } \\
\text { aumentar tanto a força muscular quan- } \\
\text { to a velocidade de processamento de } \\
\text { informações no cérebro. Mas o efeito } \\
\text { positivo do exercício é especialmente } \\
\text { associada com o lado direito do cére- } \\
\text { bro ou da mão esquerda. }\end{array}$ \\
\hline $\begin{array}{l}\text { (ECKNER } \\
\text { et al., 2011) }\end{array}$ & 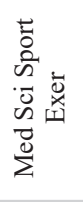 & $\begin{array}{l}26 \\
\mathrm{M} \mathrm{e} \mathrm{F} \\
26-27\end{array}$ & $\begin{array}{l}\text { Adultos saudáveis } \\
\text { praticantes de exer- } \\
\text { cícios e atividades } \\
\text { físicas. }\end{array}$ & $\begin{array}{l}\text { Não usou } \\
\text { computador } \\
\text { (régua adap- } \\
\text { tada pelo } \\
\text { autor) }\end{array}$ & $\begin{array}{l}\text { Determinar se um teste clí- } \\
\text { nico de tempo de reação } \\
\text { simples poderia prever uma } \\
\text { resposta de proteger a cabe- } \\
\text { ça em uma ação relacionada } \\
\text { ao desporto. }\end{array}$ & A & $\begin{array}{l}\text { Resultados revelam que TRsimples } \\
\text { está fortemente correlacionado com a } \\
\text { tarefa funcional projetada para simular } \\
\text { uma resposta natural de proteção da } \\
\text { cabeça em um ambiente relacionado } \\
\text { com o desporto. }\end{array}$ \\
\hline $\begin{array}{l}\text { (KA- } \\
\text { MINSKI; } \\
\text { GROFF; } \\
\text { GLUT- } \\
\text { TING, } \\
\text { 2009) }\end{array}$ & 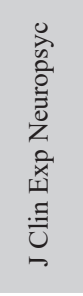 & $\begin{array}{l}25 \\
\mathrm{M} \mathrm{e} \mathrm{F} \\
18^{*}\end{array}$ & Colegiais ativos. & ANAM & $\begin{array}{l}\text { Examinar a estabilidade } \\
\text { das medidas decorrentes } \\
\text { da bateria de testes com- } \\
\text { putadorizados Automated } \\
\text { Neuropsychological Assess- } \\
\text { ment Metric (ANAM) para } \\
\text { determinar em que ponto os } \\
\text { resultados dos testes se esta- } \\
\text { bilizam. }\end{array}$ & A & $\begin{array}{l}\text { Os resultados mostram que dois perío- } \\
\text { dos de tempo são suficientes para obter } \\
\text { resultados estáveis. }\end{array}$ \\
\hline $\begin{array}{l}\text { (KELLY et } \\
\text { al., 2012) }\end{array}$ & 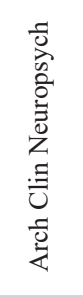 & $\begin{array}{c}212 \\
\mathrm{M} \\
22-31\end{array}$ & $\begin{array}{l}\text { Soldados norte- } \\
\text {-americanos. }\end{array}$ & ANAM & $\begin{array}{l}\text { Avaliar a validade do Au- } \\
\text { tomated Neuropsycholo- } \\
\text { gical Assessment Metrics } \\
\text { (ANAM) em ambiente de } \\
\text { combate para avaliar contu- } \\
\text { sões na cabeça. }\end{array}$ & A & $\begin{array}{l}\text { Resultados demonstram que ANAM, } \\
\text { e, particularmente o tempo de reação } \\
\text { simples é mais eficaz que a tradicional } \\
\text { brief sports medicine neuropsycho- } \\
\text { logical battery na diferenciação dos } \\
\text { participantes lesionados e não lesiona- } \\
\text { dos no ambiente de combate, quando } \\
\text { administrado } 72 \text { horas após a lesão na } \\
\text { cabeça. }\end{array}$ \\
\hline $\begin{array}{l}\text { (KIDA; } \\
\text { ODA; } \\
\text { MATSU- } \\
\text { MURA; } \\
\text { 2005) }\end{array}$ & 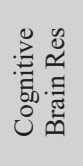 & $\begin{array}{c}193 \\
\mathrm{M} \\
20-22\end{array}$ & $\begin{array}{l}\text { Jogadores de bei- } \\
\text { sebol, jogadores de } \\
\text { tênis e estudantes } \\
\text { sedentários. }\end{array}$ & $\begin{array}{l}\text { Não mencio- } \\
\text { nou }\end{array}$ & $\begin{array}{l}\text { Avaliar os efeitos da expe- } \\
\text { riência no beisebol sobre } \\
\text { tarefas de tempo de reação } \\
\text { simples e Go/NoGo. }\end{array}$ & $\mathrm{C}$ & $\begin{array}{l}\text { O tempo de reação simples não é um } \\
\text { preditor particularmente preciso de ex- } \\
\text { periência, desempenho ou sucesso nos } \\
\text { esportes. }\end{array}$ \\
\hline $\begin{array}{l}\text { (VANTTI- } \\
\text { NEN et al., } \\
2010 \text { ) }\end{array}$ & 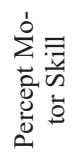 & $\begin{array}{c}428 \\
\mathrm{M} \\
10-16\end{array}$ & $\begin{array}{l}\text { Jogadores elite e } \\
\text { subelite de futebol e } \\
\text { não jogadores. }\end{array}$ & $\begin{array}{l}\text { Wayne Sac- } \\
\text { cadic Fixator } \\
\text { board (Way- } \\
\text { ne Enginee- } \\
\text { ring, USA) }\end{array}$ & $\begin{array}{l}\text { Acompanhar o desenvolvi- } \\
\text { mento geral de habilidades } \\
\text { motoras e de percepção em } \\
\text { jogadores de futebol e não } \\
\text { jogadores. }\end{array}$ & $\mathrm{C}$ & $\begin{array}{l}\text { As medidas gerais de habilidades mo- } \\
\text { toras e perceptivas melhoraram em } \\
\text { crianças mais velhas, de } 10 \text { a } 16 \text { anos, } \\
\text { com a melhora relativamente maior } \\
\text { nas tarefas mais exigentes. }\end{array}$ \\
\hline
\end{tabular}

$\overline{\mathrm{M}=\text { masculino; } \mathrm{F}=\text { feminino; }}$ *=média de idade dos participantes; ANAM=Automated Neuropsychological Assessment Metrics; CRI=Concussion Resolution Index; Id=Identificação do agrupamento dos temas da discussão, sendo: a) Uso do TRSimples para avaliação da concussão cerebral; b) Efeito do exercício físico sobre o desempenho no TRSimples; c) Níveis de prática e comparações do TRSimples de atletas e não atletas; e d) Fatores que influenciam o TRSimples.

Houve predominância de estudos com estudantes universitários e as modalidades investigadas foram variadas. O total de participantes dos estudos foi de 1.065 , variando entre 15 a 428 sujeitos (média de 118,3). Todos os estudos envolveram homens e quatro deles também avaliaram as mulheres. Todas as pesquisas envolveram participantes até 31 anos.

Tabela 3: Características dos estudos incluídos para a seleção por "Tempo de reação simples" e "Exercise" ou "Physical activity" (ordenados alfabeticamente)

\begin{tabular}{|c|c|c|c|c|c|c|c|}
\hline Referência & 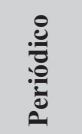 & $\begin{array}{c}\mathrm{N} \\
\text { Sexo } \\
\text { Idade }\end{array}$ & População & Instrumento & Tema & Cat & Resultado \\
\hline $\begin{array}{l}\text { (ALLEN et } \\
\text { al., 2003) }\end{array}$ & 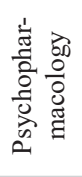 & $\begin{array}{c}72 \\
\mathrm{M} \\
18-34\end{array}$ & $\begin{array}{c}\text { Homens saudá- } \\
\text { veis }\end{array}$ & $\begin{array}{l}\text { MacLabo- } \\
\text { ratory task } \\
\text { (Chute 1994) }\end{array}$ & $\begin{array}{l}\text { Avaliar o desempenho em } \\
\text { tarefas cognitivas e moto- } \\
\text { ras em indivíduos saudáveis } \\
\text { com dano muscular. }\end{array}$ & B & $\begin{array}{l}\text { Hydrocodone além de ibuprofeno não foi } \\
\text { associada a deterioração da cognição com- } \\
\text { plexa, mas estava relacionado com decrés- } \\
\text { cimos em tarefas que envolvem a coorde- } \\
\text { nação simples olho-mão. }\end{array}$ \\
\hline $\begin{array}{l}\text { (ANDO et } \\
\text { al., 2009) }\end{array}$ & 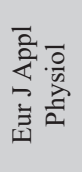 & $\begin{array}{c}12 \\
\mathrm{M} \\
25^{*}\end{array}$ & Homens & $\begin{array}{l}\text { Qtec Co., } \\
\text { Ltd, Japan }\end{array}$ & $\begin{array}{l}\text { Testar se o aumento da dis- } \\
\text { ponibilidade de oxigênio } \\
\text { afeta o tempo de reação sim- } \\
\text { ples com estímulo visual pe- } \\
\text { riférico durante o exercício. }\end{array}$ & $\mathrm{D}$ & $\begin{array}{l}\text { Resultados sugerem que o aumento da dis- } \\
\text { ponibilidade de oxigênio durante o exercí- } \\
\text { cio tem efeitos benéficos sobre o desempe- } \\
\text { nho perceptual. }\end{array}$ \\
\hline
\end{tabular}


(ANDO;

YAMADA;

KOKUBU, 2010)
10

M

25

Homens

Qtec Co.,

Ltd, Japan

Testar se a diminuição da oxigenação cerebral compromete a capacidade de um indivíduo para responder aos estímulos visuais periféricos durante o exercício.

\begin{tabular}{|c|c|c|c|c|c|}
\hline $\begin{array}{l}\text { (DRAPER; } \\
\text { MC- } \\
\text { MORRIS; } \\
\text { PARKER, } \\
\text { 2010) }\end{array}$ & 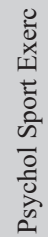 & $\begin{array}{c}12] \\
\mathrm{M} \\
31^{*}\end{array}$ & $\begin{array}{c}\text { Homens fisica- } \\
\text { mente ativos }\end{array}$ & Próprio & $\begin{array}{l}\text { Comparar a diferença no } \\
\text { efeito do exercício agudo } \\
\text { de curta duração sobre o de- } \\
\text { sempenho de tarefas de tem- } \\
\text { po de reação visual simples } \\
\text { e escolha. }\end{array}$ \\
\hline
\end{tabular}

Descobrimos uma forte correlação entre o aumento no tempo pré-motor e diminuição da oxigenação cerebral, sugerindo que

D o aumento no tempo premotor durante o exercício está associada com diminuição da oxigenação cerebral. Assim, o exercício em grandes altitudes pode comprometer o desempenho perceptual visual.

Os resultados suportam a hipótese de que o exercício afeta significativamente os tempos de reação simples e de escolha de forma diferente. $\mathrm{O}$ efeito da interação mos-

B trou que o tempo de reação simples, não foi significativamente afetado pelo aumento da intensidade do exercício, enquanto o tempo de reação de escolha mostrou uma redução linear com o aumento da intensidade do exercício.

\begin{tabular}{|c|c|c|c|c|}
\hline $\begin{array}{l}\text { (ELLEM- } \\
\text { BERG; } \\
\text { ST-LOUIS- } \\
\text {-DESCHE- } \\
\text { NES, 2010) }\end{array}$ & 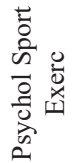 & $\begin{array}{c}72 \\
\mathrm{M} \\
7-10\end{array}$ & $\begin{array}{l}\text { Meninos fisica- } \\
\text { mente ativos }\end{array}$ & Matlab \\
\hline
\end{tabular}

Determinar se curtos períodos de exercício físico melhoraram a função cognitiva em crianças e se este efeito varia com a idade durante o desenvolvimento.

\begin{tabular}{|c|c|c|c|c|}
\hline $\begin{array}{l}\text { (GON- } \\
\text { ZALEZ; } \\
\text { TUBIO; } \\
\text { SANCHEZ, } \\
2011)\end{array}$ & 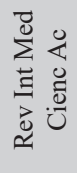 & $\begin{array}{c}50 \\
F \\
52-87\end{array}$ & $\begin{array}{l}\text { Mulheres chile- } \\
\text { nas que pratica- } \\
\text { vam exercicio } \\
\text { aerobio } 3 \mathrm{x} \text { por } \\
\text { semana } 60 \mathrm{~min} \text {. }\end{array}$ & $\begin{array}{c}\text { Software E- } \\
\text {-prime } 1.2\end{array}$ \\
\hline $\begin{array}{l}\text { (KVELDE } \\
\text { et al., 2010) }\end{array}$ & 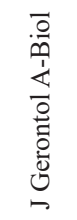 & $\begin{array}{c}280 \\
\mathrm{M} \text { e F } \\
62-95\end{array}$ & $\begin{array}{c}\text { Moradores de } \\
\text { Sidney, Austrália }\end{array}$ & $\begin{array}{c}\text { Não mencio- } \\
\text { nou }\end{array}$ \\
\hline
\end{tabular}

Desenvolver um programa de exercícios durante 8 semanas para melhorar a capacidade de resposta e mitigar os efeitos do envelhecimento cognitivo.

Investigar por que as pessoas deprimidas são mais propensos a cair, explorandoa relação entre os sintomas depressivos auto-relatados e desempenho em tarefas cognitivas.

Resultados indicam que uma única e curta sessão de atividade aeróbica de intensida-

B de moderada melhora significativamente o tempo de resposta para as tarefas simples e de escolha.

A realização de exercícios específicos re-

B duz o tempo de reação em situações complexas.

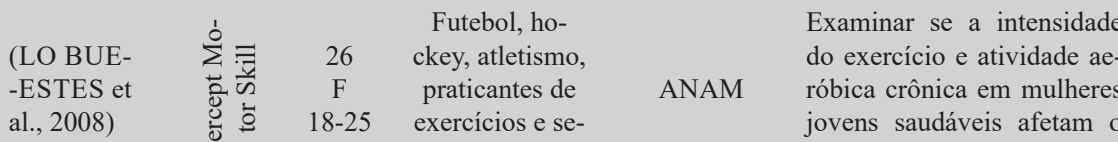

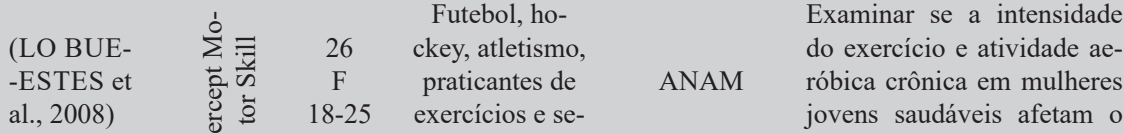
jovens saudáveis afetam
desempenho cognitivo.

Análise mostrou que o tempo de reação B simples foi mais rápido nas mulheres ativas do que nas sedentárias, mas não foi afetada pelo exercício.

Determinar se um programa

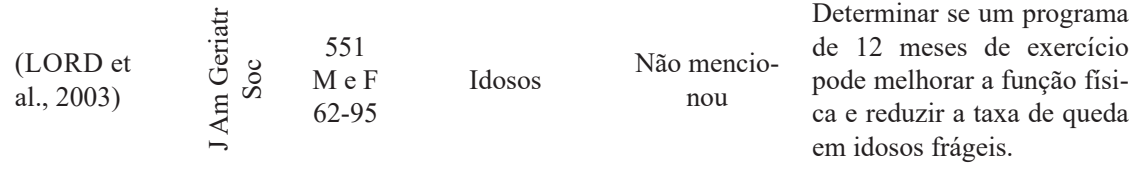

Eficácia do exercício como uma estratégia de prevenção de quedas, mostrando que o B exercício baseado em grupo pode produzir benefícios no que diz respeito à manutenção do funcionamento físico e reduzindo a frequência de quedas em idosos frágeis.

\begin{tabular}{|c|c|c|c|c|}
\hline $\begin{array}{l}\text { (MURRAY; } \\
\text { RUSSO- } \\
\text { NIELLO, } \\
\text { 2012) }\end{array}$ & 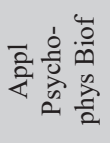 & $\begin{array}{c}120 \\
\mathrm{M} \mathrm{e} \mathrm{F} \\
18-25\end{array}$ & $\begin{array}{l}\text { Voluntários Uni- } \\
\text { versidade USA }\end{array}$ & Próprio \\
\hline $\begin{array}{l}\text { (NEWSON; } \\
\text { KEMPS, } \\
2008)\end{array}$ & 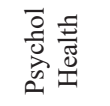 & $\begin{array}{c}96 \\
\mathrm{M} \\
18-65\end{array}$ & Mulheres & $\begin{array}{c}\text { Não mencio- } \\
\text { nou }\end{array}$ \\
\hline
\end{tabular}

Examinar a relação entre a atividade física e a função cognitiva em participantes ativos e não-ativos.

Houve melhora significativa do desempe-

B nho cognitivo em tarefas com maior componente cognitivo e perceptual.

Investigar a associação entre aptidão cardiorrespiratória e envelhecimento cognitivo.

Os adultos mais jovens realizam todas as

B tarefas cognitivas em um nível mais elevado do que os adultos mais velhos.

Examinar o efeito da corrida

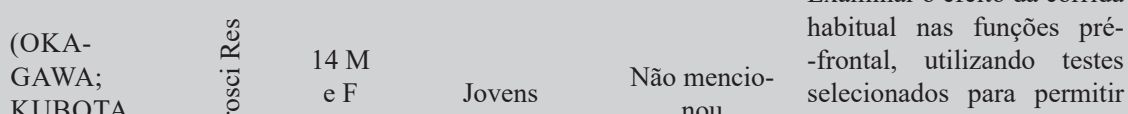

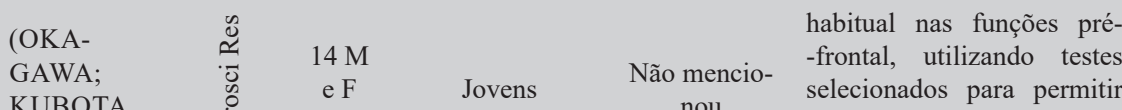
$\begin{array}{lllll}\text { KUBOTA, } & \text { e F } & \text { Jovens } & \text { nou } & \text { selecionados para permitir } \\ & 0 & 27^{*} & & \text { a detecção de alterações }\end{array}$ funcionais no córtex pré-frontal.

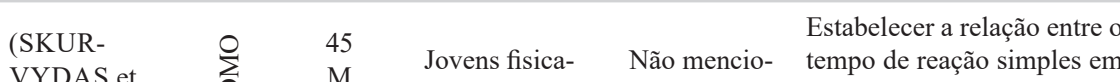
VYDAS et al., 2009) $18-22$ mente ativos jovens adultos e o seu índice de massa corporal.
B A corrida habitual melhora o desempenho em uma tarefa de ramificação pré-frontal.

Participantes do grupo com maior índice de

D massa corporal reagiram significativamente mais lento do que os outros. 


\begin{tabular}{|c|c|c|c|c|c|c|c|}
\hline $\begin{array}{l}\text { (SKUR- } \\
\text { VYDAS et } \\
\text { al., 2010) }\end{array}$ & 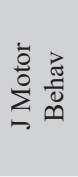 & $\begin{array}{c}11 \\
\mathrm{M} \\
24^{*}\end{array}$ & $\begin{array}{l}\text { Homens fisica- } \\
\text { mente ativos }\end{array}$ & $\begin{array}{c}\text { Não mencio- } \\
\text { nou }\end{array}$ & $\begin{array}{l}\text { Examinar o efeito da fadiga } \\
\text { muscular causada pelo exer- } \\
\text { cício sobre o desempenho } \\
\text { cognitivo (Pernas). }\end{array}$ & B & $\begin{array}{l}\text { Exercício aumentou da variabilidade de } \\
\text { torque isométrico submáximo e a variabili- } \\
\text { dade intra-individual no desempenho cog- } \\
\text { nitivo, mas não afetou o tempo de reação, } \\
\text { um índice de desempenho cognitivo. }\end{array}$ \\
\hline $\begin{array}{l}\text { (SHIPLEY } \\
\text { et al., 2007) }\end{array}$ & 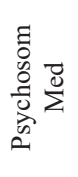 & $\begin{array}{l}3.802 \\
\mathrm{M} \mathrm{e} \mathrm{F} \\
18-94\end{array}$ & Adultos & $\begin{array}{c}\text { Não mencio- } \\
\text { nou }\end{array}$ & $\begin{array}{l}\text { Examinar a associação entre } \\
\text { a alteração no tempo de rea- } \\
\text { ção e desempenho cognitivo } \\
\text { ao longo de } 7 \text { anos. }\end{array}$ & D & $\begin{array}{l}\text { Resultados sugerem que o aumento da mé- } \\
\text { dia e a variabilidade do TRS e diminuição } \\
\text { no desempenho da memória e raciocínio } \\
\text { estão associados com um aumento do risco } \\
\text { de mortalidade e morte por diversas causas. }\end{array}$ \\
\hline $\begin{array}{l}\text { (SMILEY- } \\
\text {-OYEN et } \\
\text { al., 2008) }\end{array}$ & 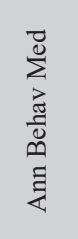 & $\begin{array}{c}57 \\
\mathrm{M} \mathrm{e} \mathrm{F} \\
65-79\end{array}$ & Idosos & $\begin{array}{c}\text { Não mencio- } \\
\text { nou }\end{array}$ & $\begin{array}{l}\text { Investigar se as mudanças } \\
\text { no desempenho nas tarefas } \\
\text { cognitivas foram relaciona- } \\
\text { das com alterações na capa- } \\
\text { cidade aeróbica. }\end{array}$ & B & $\begin{array}{l}\text { O exercício aeróbico em adultos mais ve- } \\
\text { lhos podem ter um efeito benéfico sobre o } \\
\text { desempenho de tarefas mais rápidas que } \\
\text { dependem fortemente de controle executi- } \\
\text { vo. Melhorias na capacidade aeróbica não } \\
\text { parece ser um pré-requisito para esse efeito } \\
\text { benéfico. }\end{array}$ \\
\hline
\end{tabular}

$\overline{\mathrm{M}}=$ masculino; $\mathrm{F}=$ feminino; *=média de idade dos participantes; ANAM=Automated Neuropsychological Assessment Metrics; Id=Identificação do agrupamento dos temas da discussão, sendo: a) Uso do TRSimples para avaliação da concussão cerebral; b) Efeito do exercício físico sobre o desempenho no TRSimples; c) Níveis de prática e comparações do TRSimples de atletas e não atletas; e d) Fatores que influenciam o TRSimples.

A análise dos artigos que fizeram uso de um teste de TRSimples relacionado com exercício ou atividade física resultou em 16 artigos e são apresentados na Tabela 3. Os temas abordados nos estudos também foram variados, sendo que sete avaliaram o efeito do exercício sobre as funções cognitivas (ALLEN et al., 2003; OKAGAWA; KUBOTA, 2004; LO BUE-ESTES et al., 2008; DRAPER; MCMORRIS; PARKER, 2010; ELLEMBERG; ST-LOUIS-DESCHENES 2010; SKURVYDAS et al., 2010; MURRAY; RUSSONIELLO, 2012) ou a influência do exercício em idosos (LORD et al., 2003; SMILEY-OYEN et al., 2008); GONZALEZ; TUBIO; SANCHEZ, 2011) sendo todos com instrumentos distintos.

As populações participantes foram variadas, com um total de 4.867, variando entre 10 a 3.802 sujeitos (média de 486,7). A maioria dos artigos envolveram homens $(n=14)$ e sete deles também avaliaram as mulheres (dentre estes, dois avaliaram apenas mulheres). A faixa de idade variou de 7 a 95 anos.

Nos 36 artigos analisados, o instrumento mais utilizado foi o Automated Neuropsychological Assessment Metrics - ANAM $(n=6)$ que consiste de uma série de testes para avaliar diferentes funções cognitivas (BROWN; GUSKIEWICZ; BLEIBERG, 2007) e foi projetado para detectar a velocidade e a precisão da atenção, memória e capacidade de raciocínio. Dez artigos não mencionaram o software utilizado e quatro artigos não utilizaram software para o teste de TRSimples, mas sim outros instrumentos: a) um temporizador de reação elétrico para a medição de TR de estímulos auditivo e visual (CHANDRA et al., 2010); Newtest 2000 Reaction Time Apparatus que consiste de um painel de reação com duas luzes de LED e dois botões e um painel de controle com cronômetro e programação dos botões (DANE; HAZAR; TAN, 2008); c) clinical reaction time apparatus criado pelo próprio autor (ECKNER et al., 2011); d) Wayne Saccadic Fixator board que consiste de um painel com botões que devem ser pressionados no momento em que se acendem (VANTTINEN et al., 2010).

\section{Discussão}

Este estudo teve como objetivo analisar a produção científica relacionada ao uso de testes de tempo de reação simples com estímulo visual (TRSimples) relacionado à Educação Física para apresentar uma síntese da evidência empírica disponível sobre a eficácia do uso deste tipo de teste em atletas, praticantes de exercício ou atividade física, buscando identificar a contribuição potencial destes testes para o profissional da Educação Física e para a Psicologia do Esporte e do Exercício.

A seguir são discutidos os artigos revisados, separando-os por tema.

\section{(a) Uso do TRSimples para avaliação da concussão cere- bral}

Os artigos incluídos neste tema compreendem estudos de avaliação do uso de instrumentos com testes de TRSimples para avaliação das lesões após concussão cerebral (causado por lesão na cabeça durante a prática esportiva), condição em que os artigos abordam a consciência do problema, prevenção e tratamento destinados, principalmente, aqueles envolvidos na prática de esportes de alto risco. Os benefícios potenciais de testes de TRSimples mencionados nesses artigos são apresentados em primeiro lugar, seguido de resumos dos artigos, incluindo os resultados da avaliação sobre a eficácia real destes testes.

Erlanger et al. (2003) desenvolveram e validaram uma bateria de testes computadorizados o Concussion Resolution Index (CRI), concebido para acompanhar a resolução dos sintomas após concussão cerebral relacionada aos esportes, afirmando ser um método válido e confiável para determinar mudanças na velocidade psicomotora e na velocidade de processamento de informações para acompanhar lesões na cabeça relacionada aos esportes. Esta bateria foi utilizada por Bailey et al. (2010) para avaliar o efeito do estresse psicológico sobre o desempenho neurocognitivo após pancada na cabeça, revelando correlações significativas entre os aspectos somáticos como esquizofrenia, preocupação, depressão, ansiedade, abuso de drogas e ideias suicidas e o desempenho no TR, e quando examinados categoricamente, os participantes com ideias suicidas apresentaram um TR mais lento.

Outra sequela comum e importante de concussão cerebral é o prolongamento do tempo de reação (ECKNER 
et al., 2011). Utilizando o instrumento "clinical reaction time apparatus" já validado anteriormente, Eckner, Kutcher e Richardson, (2011) propõem o uso deste novo instrumento em uma amostra de 102 atletas de esporte de alto impacto (futebol americano, futebol e luta) para determinar a confiabilidade teste-reteste medido ao longo de sucessivas temporadas e comparar estes resultados com o software CogState Sport (CogState Ltd, Melbourne, Australia); Eckner et al., (2011) também propõem o seu uso em comparação com o desempenho motor em uma tarefa de proteger a cabeça interceptando um objeto lançado, encontrando forte correlação do TRSimples com a tarefa funcional projetada para simular uma resposta natural de proteção da cabeça em um ambiente relacionado com o desporto.

Como o TRSimples tem se mostrado uma variável sensível à avaliação de contusões na cabeça relacionados com o desporto, Kaminski, Groff e Glutting, (2009) utilizaram uma amostra de estudantes colegiais ativos em atividades de recreação aplicando teste-reteste com o software ANAM, para determinar o número de execuções em que o teste neuropsicológico se estabiliza. Os autores afirmaram que dois períodos de tempo são suficientes para obter resultados estáveis sendo possível aplicar duas medidas para comparação e follow-up.

O estudo de Kelly et al. (2012) utilizaram uma amostra de soldados norte-americanos no Iraque com concussão cerebral aguda (72 horas após lesão), demonstrando que o tempo de reação simples foi mais eficaz que a bateria tradicional "brief sports medicine neuropsychological" na diferenciação dos participantes lesionados e não lesionados no ambiente de combate, quando administrado 72 horas após a lesão na cabeça.

Embora os especialistas discordem nas recomendações específicas sobre a avaliação da gravidade do estado de um atleta após concussão cerebral e os parâmetros de retorno ao esporte, eles geralmente aceitam que os atletas não devem ser expostos à prática regular de suas atividades esportivas até que todos os sintomas de concussão cerebral tenham sido resolvidos (ERLANGER et al., 2003; SCHMIDT et al., 2012). Isso foi reforçado pelo pior desempenho observado em atletas com dores de cabeça pós-traumática, sugerindo que a dor de cabeça, concussão cerebral e dor geral podem ter algum efeito sobre a função neurocognitiva, reforçando a importância de que os traumas sejam assintomáticos antes que o atleta volte a jogar (REGISTER-MIHALIK et al., 2007).

Como os testes neurocognitivos informatizados demandam muito tempo e normalmente envolvem altos custos, Schmidt et al. (2012) sugerem que os profissionais ligados ao esporte, sem recursos e tempo adequados, podem identificar na sua maioria, as mesmas deficiências neurocognitivas utilizando valores normativos que permitem ignorar o longo processo de estabelecer medidas iniciais individualizadas como parte de um programa de avaliação de concussão cerebral multifacetada.

Embora não fosse o objetivo principal do estudo, Brown, Guskiewicz e Bleiberg, (2007) avaliaram os resultados do ANAM e não observaram diferenças no total de pontos com base no histórico de concussão cerebral, afirmando que os potenciais efeitos interativos de concussão cerebral anterior, distúrbio de aprendizagem e inteligência dificulta- ram as comparações, principalmente pelo fato de que alguns atletas deste estudo podem ter tido contusões que não foram diagnosticados.

Profissionais ligados ao esporte se deparam com a difícil tarefa de avaliar e gerir os sintomas resultantes de concussões cerebrais relacionadas com o desporto (SCHMIDT et al., 2012). O mais importante é a necessidade de identificar os atletas que experimentam sofrimento psicológico significativo e proporcionar-lhes oportunidades de tratamento para amenizar os sintomas da concussão cerebral (ERLANGER et al., 2003) geralmente identificada por cognição prejudicada, falta de equilíbrio além de outros sintomas subjetivos (KELLY et al., 2012).

\section{(b) Efeito do exercício físico sobre o desempenho no TR- Simples}

Neste tema foram incluídos os artigos que avaliaram especificamente o efeito do exercício físico sobre o desempenho no TRSimples.

Técnicas para avaliação das atividades no cérebro durante o exercício têm indicado evidências de que o exercício está associado com mudanças profundas nas regiões motora, sensorial e autonômica do cérebro. Aumentos acentuados na ativação ocorrem em estruturas neurais responsáveis por gerar os padrões motores que sustentam a atividade física. Em particular, o córtex motor primário e secundário, gânglios basais, cerebelo, mesencéfalo e diversos núcleos do tronco cerebral, vias motoras, assim como vários núcleos talâmicos estão envolvidos. Tomados em conjunto, essas regiões neurais representam uma percentagem substancial da massa total do cérebro, confirmando que o exercício físico requer a ativação neuronal maciça em um grande número de estruturas neurais através de todo o cérebro (DIETRICH; AUDIFFREN, 2011), sendo que a medição do tempo de reação tem se estabelecido como um meio eficaz para quantificar a taxa em que são realizados os processos mentais (GONZALEZ; TUBIO; SANCHEZ, 2011).

Apesar de parecer clara a influência do exercício sobre as atividades neurocognitivas, ainda existem questões a serem respondidas como a diferença no efeito do exercício agudo de curta duração (LO BUE-ESTES et al., 2008; DRAPER; MCMORRIS; PARKER, 2010) ou do exercício físico agudo durante o desenvolvimento de crianças (ELLEMBERG; ST-LOUIS-DESCHENES, 2010), também pouco se sabe sobre a extensão em que a fadiga e dano muscular afetam a função cognitiva (ALLEN et al., 2003; SKURVYDAS et al., 2010).

Lo Bue-Estes et al. (2008) verificaram que a intensidade do exercício e a quantidade de atividade aeróbia crônica em mulheres jovens saudáveis afeta o desempenho cognitivo, porém, o TRSimples não foi afetado pelo exercício (mesmo resultado encontrado por Okagawa e Kubota, (2004) em jovens saudáveis praticantes de cooper), mas foi significativamente mais rápido para o grupo ativo do que para o grupo sedentário em pré e pós-exercício, bem como após a recuperação. O problema reside sobre a variabilidade entre os estudos sobre múltiplos fatores que podem afetar o resultado, inclusive da população (idade, sexo, nível de atividade, exercício, tipo, duração, intensidade), tipo de variável cognitiva (simples ou complexa), modalidade do teste cogni- 
tivo (caneta e papel, automatizado, verbal) e tempo de testes cognitivos (antes, durante ou após o exercício) (LO BUE-ESTES et al., 2008; MURRAY; RUSSONIELLO, 2012).

No estudo de Draper, Mcmorris e Parker (2010) os resultados suportam a hipótese de que o exercício afeta significativamente o TRSimples e de escolha de forma diferente pelo exercício agudo, de curta duração de diferentes intensidades. $\mathrm{O}$ efeito da interação mostrou que o TRSimples, não foi significativamente afetado pelo aumento da intensidade do exercício, enquanto o tempo de reação de escolha mostrou uma redução linear com o aumento da intensidade. Resultado semelhante foi reportado por Murray e Russoniello (2012) onde o impacto maior do exercício foi observado nos testes com maior exigência cognitiva em jovens universitários ativos e não ativos.

Embora os artigos tenham sugerido que uma sessão aguda de exercício físico de intensidade e duração moderada melhora a função cognitiva em adultos, muito pouco se sabe sobre os efeitos de uma única sessão de exercício físico sobre o funcionamento cognitivo durante o desenvolvimento de crianças. Em função disso Ellemberg (2010) realizou um estudo para determinar se os curtos períodos de exercício físico melhoraram a função cognitiva em crianças e se este efeito variava com a idade durante o desenvolvimento. Os resultados indicaram que uma única e curta sessão de atividade aeróbica de intensidade moderada melhorou significativamente o tempo em testes de TRSimples e de escolha.

No estudo de Skurvydas et al. (2010), o exercício aumentou a variabilidade de torque isométrico submáximo e a variabilidade intraindividual no desempenho cognitivo, mas não afetou o TR, um índice de desempenho cognitivo. Porém, este foi o único artigo sobre mudanças de tempo de reação após o dano muscular induzido pelo exercício para as pernas, os outros artigos envolveram o controle de movimentos da mão. Mesmo o artigo de Allen et al. (2003) onde os autores também avaliaram o desempenho no TRSimples após dano muscular induzido pelo exercício para as pernas, o TRSimples foi executado com as mãos.

Outras conclusões reportadas nos artigos indicaram que, para o TR mais rápido, menor foi a variabilidade da frequência cardíaca, o que sugere que a modulação simpática superior foi relacionada com respostas rápidas (LUFT; TAKAS; DARBY, 2009) e que o efeito positivo do exercício foi especialmente associado com o lado direito do cérebro ou da mão esquerda (DANE; HAZAR; TAN, 2008).

A prática da atividade física e de exercícios também foi analisada em pessoas idosas (LORD et al., 2003; NEWSON; KEMPS, 2008); SMILEY-OYEN et al., 2008); GONZALEZ; TUBIO; SANCHEZ, 2011), pois estudos indicam que os efeitos do envelhecimento sobre a velocidade de processamento cognitivo foram mais baixos nos indivíduos ativos. Em função disso, autores recomendaram a inclusão de atividades físicas e exercício nos diferentes programas com idosos (GONZALEZ; TUBIO; SANCHEZ 2011), que isso pode prevenir quedas e manter o funcionamento físico em idosos (LORD et al., 2003) e que pode ajudar a proteger contra o declínio cognitivo associado ao envelhecimento (NEWSON; KEMPS, 2008). Contraditoriamente, o estudo de Smiley-Oyen et al. (2008) afirmou que o treinamento físico não influencia o desempenho em tarefas que exigem relativamente pouco controle executivo como o TRSimples.

\section{(c) Níveis de prática e comparações do TRSimples de atletas e não atletas}

Neste tema foram agrupados os estudos que trataram especificamente da relação do desempenho no TRSimples de atletas e não atletas.

Estudos buscam entender as habilidades cognitivas de atletas comparando seu desempenho aos não atletas, pois, como são reportados os benefícios do exercício, é esperado que o envolvimento em longo prazo no esporte altere as atividades neurais ou estruturais e funções cerebrais, reforçando as capacidades cognitivas como resultado das demandas específicas de determinados esportes (CHAN et al., 2011).

A prática de esportes desenvolveu a velocidade dos sistemas perceptuais e motores, embora a prática de longo prazo no esporte não melhorou as habilidades de TRSimples, melhorou apenas as habilidades em tarefas mais complexas (KIDA; ODA; MATSUMURA, 2005; BAUR et al., 2006; VANTTINEN et al., 2010; CHAN et al., 2011; COJOCARIU, 2011). Estes resultados, foram confirmados nos estudos que compararam o desempenho de não atletas com atletas de beisebol e tênis (KIDA; ODA; MATSUMURA, 2005), esgrima (CHAN et al., 2011) e futebol (VANTTINEN et al., 2010); como também entre pilotos de corrida e praticantes de esporte recreativo (corrida, natação, ciclismo, basquetebol ou futebol) (BAUR et al., 2006).

O estudo de Chaddock et al. (2011) buscou investigar se a prática do esporte facilita a execuções de atividades da vida diária, em uma tarefa que exige o processamento de várias informações, como o atravessar uma rua, sugerindo que os atletas apresentaram desempenho superior quando comparados aos não atletas.

O TRSimples, mesmo quando comparado entre atletas de mesma modalidade, mas com níveis distintos de prática, não apresentou diferenças significativas (no $Q$ wan $K i$ $D o$ ), embora atletas mais experientes apresentaram tempos menores (COJOCARIU, 2011). Diferentemente, Loureiro e De Freitas, (2012) concluíram que os jogadores de badminton de alto nível reagiram mais rapidamente a um estímulo visual do que jogadores de nível inferior durante um teste de apontar um alvo. Yotani et al. (2011) demonstraram que foi possível melhorar o processo nervoso central para melhorar o TRSimples, pois indivíduos que tiveram um maior tempo antes do treino mostraram uma maior redução no tempo após o treinamento.

\section{(d) Fatores que influenciam o TRSimples}

Neste tema são apresentados os artigos selecionados que investigaram alguns fatores que podem influenciar o desempenho em um teste de TRSimples.

O estudo de Brown, Guskiewicz e Bleiberg, (2007) procurou investigar os efeitos de fatores que influenciaram o TRSimples como sexo, índice escolar (SAT), estado de alerta e tipo de esporte praticado, para estabelecer dados de referência preliminares para atletas colegiais em vários esportes, encontrando diferenças entre todos estes fatores.

Skurvydas et al. (2009) exploraram a possível existência de uma relação entre TRSimples e IMC, e os resultados demonstraram que não houve diferença significativa no TRSimples entre os grupos com diferentes IMC. No entan- 
to, o grupo de participantes com um nível mais elevado de IMC produziu um TRSimples significativamente mais longo, quando considerados os resultados em intervalos modais. Resultado semelhante foi reportado por Gentier et al. (2013), em um estudo comparativo do desempenho em tarefas de tempo de reação simples e de escolha entre crianças obesas e de peso saudável, os autores concluíram que as crianças obesas foram mais lentas em funções executivas e de tomada de decisão, a fim de planejar e controlar o movimento.

Kvelde et al. (2010) buscaram investigar por que pessoas deprimidas são mais propensas a cair, explorando a relação entre os sintomas depressivos autorrelatados e desempenho em testes cognitivos, sendo que os resultados sugerem que o humor deprimido autorelatado foi relacionado ao desempenho reduzido em testes cognitivos.

A influência do aumento dos níveis de oxigênio no cérebro sobre o desempenho no TRSimples durante o exercício foi apresentado em dois artigos (ANDO et al., 2009; ANDO; YAMADA; KOKUBU, 2010), sugerindo que o aumento da disponibilidade de oxigênio durante o exercício tem efeitos benéficos sobre o desempenho perceptual, sendo esta uma informação relevante para o exercício em grandes altitudes, onde existe uma diminuição da oxigenação cerebral, e isto tem implicações para o desempenho perceptual visual durante o exercício.

O efeito do calor sobre o exercício físico moderado foi avaliado em estudantes de pós-graduação saudáveis, com resultados indicando uma diminuição do TR após o exercício, mas um aumento acentuado quando o exercício foi realizado em temperaturas elevadas (CHANDRA et al., 2010).

O estudo de Shipley et al. (2007) acompanhou 3.802 adultos por 7 anos, avaliando dados sociodemográficos, estilo de vida (que incluiu o nível de atividade física), saúde e informação fisiológica. Os resultados dos testes cognitivos com declínios na média e variabilidade do TRSimples, de escolha, a memória e o raciocínio visuo-espacial foram associados com um aumento significativo do risco de morte por todas as causas, por todas as doenças cardiovasculares, doença arterial coronariana, acidente vascular cerebral e doenças respiratórias.

\section{Conclusão}

Este estudo analisou a produção científica relacionada ao uso de testes de tempo de reação simples com estímulo visual (TRSimples), identificando a contribuição potencial deste tipo de teste de avaliação do desempenho cognitivo e a síntese de resultados empíricos relevantes.

O resultado dos estudos apontaram associações positivas entre o uso de testes de TRSimples para análises da concussão cerebral originada pela prática esportiva e apontou evidências do efeito positivo do exercício físico sobre o desempenho cognitivo. Os testes de TRSimples permitiram a avaliação de diferentes níveis de prática de atletas de mesma modalidade, de modalidades distintas e de comparação com não atletas, além de ser um indicador sensível a diversos fatores que podem influenciar o desempenho cognitivo.

A síntese apresentada nos temas centrais: "Uso do TRSimples para avaliação da concussão cerebral", "Efeito do exercício físico sobre o desempenho no TRSimples", "Níveis de prática e comparações do TRSimples de atletas e não atletas" e "Fatores que influenciam o TRSimples" proporcionam uma valiosa orientação científica da eficácia do uso de testes de TRSimples em suas diversas aplicações.

A análise realizada sugere que os testes de TRSimples apresentam benefícios potenciais para Educação Física e para a Psicologia do Esporte e do Exercício que podem influenciar positivamente os comportamentos em relação à saúde e ao exercício físico, além de melhorar as análises da aprendizagem de habilidades motoras.

\section{Referências}

ALLEN, G. J. et al. Cognitive and motor function after administration of hydrocodone bitartrate plus ibuprofen, ibuprofen alone, or placebo in healthy subjects with exercise-induced muscle damage: a randomized, repeateddose, placebo-controlled study. Psychopharmacology, v. 166, n. 3, p. 228-233, Mar 2003. ISSN 0033-3158. Disponível em: $<<$ Go to ISI $>$ ://WOS:000182202000005 >.

ANDO, S.; YAMADA, Y.; KOKUBU, M. Reaction time to peripheral visual stimuli during exercise under hypoxia. Journal of Applied Physiology, v. 108, n. 5, p. 1210-1216, May 2010. ISSN 8750-7587. Disponível em: $<<$ Go to ISI>://WOS:000277301000026 >.

ANDO, S. et al. Reaction time to peripheral visual stimuli during exercise under normoxia and hyperoxia. European Journal of Applied Physiology, v. 106, n. 1, p. 61-69, May 2009. ISSN 1439-6319. Disponível em: $<<$ Go to ISI $>: / /$ WOS:000265397400007>.

ANDRADE, A.; BELMONTE, A. P.; VIANA, M. D. S. Tempo de reação, flexibilidade e velocidade acíclica de membros inferiores de atletas de tae kwon do. Lecturas, Educación Física y Deportes, Revista Digital. Buenos Aires. 112006.

ANDRADE, A. et al. Burnout no esporte: Revisão sistemática na base ScienceDirect. Revista Kinesis, v. 30, n. 1, p. 200-215, 2012. ISSN 0102-8308 2316-5464 online.

Relação entre tempo de reação e o tempo de prática no tênis de campo. http://www.efdeportes.com/ Revista Digital. Buenos Aires. 102005.

BAAYEN, R. H. Analyzing Reaction Times. International Journal of Psychological Research, v. 3, n. 2, p. 12-28, 2010. ISSN 2011792220112084.

BAILEY, C. M. et al. The Relationship Between Psychological Distress and Baseline Sports-Related Concussion Testing. Clinical Journal of Sport Medicine, v. 20, n. 4, p. 272-277, Jul 2010. ISSN 1050-642X. Disponível em: $<<$ Go to ISI $>$ :/WOS:000279641800005 >.

BAUR, H. et al. Reactivity, stability, and strength performance capacity in motor sports. British Journal of Sports Medicine, v. 40, n. 11, p. 906-910, Nov 2006. ISSN 0306-3674. Disponível em: $<<$ Go to ISI $>$ :// WOS:000241494700005>. 
BOSCHKER, M. S. J.; BAKKER, F. C.; MICHAELS, C. F. Effect of mental imagery on realizing affordances. Quarterly Journal of Experimental Psychology Section a-Human Experimental Psychology, v. 55, n. 3, p. 775792, Aug 2002. ISSN 0272-4987. Disponível em: $<<$ Go to ISI $>$ ://WOS:000176300800004 >.

BROWN, C. N.; GUSKIEWICZ, K. M.; BLEIBERG, J. Athlete characteristics and outcome scores for computerized neuropsychological assessment: A preliminary analysis. Journal of Athletic Training, v. 42, n. 4, p. 515-523, Oct-Dec 2007. ISSN 1062-6050. Disponível em: $<<$ Go to ISI>:/WOS:000252343100012>.

CHADDOCK, L. et al. Do Athletes Excel at Everyday Tasks? Medicine and Science in Sports and Exercise, v. 43, n. 10, p. 1920-1926, Oct 2011. ISSN 0195-9131. Disponível em: $<<$ Go to ISI $>$ ://WOS:000294955800014 >.

CHAN, J. S. Y. et al. Fencing expertise and physical fitness enhance action inhibition. Psychology of Sport and Exercise, v. 12, n. 5, p. 509-514, 2011. ISSN 1469-0292. Disponível em: < http://www.sciencedirect.com/science/ article/pii/S1469029211000549>.

CHANDRA, A. M. et al. Effect of Exercise and Heat-Load on Simple Reaction Time of University Students. International Journal of Occupational Safety and Ergonomics, v. 16, n. 4, p. 497-505, 2010. ISSN 1080-3548. Disponível em: < < Go to ISI $>$ :// WOS:000285491900009>.

CHUNG, P.; NG, G. Taekwondo training improves the neuromotor excitability and reaction of large and small muscles. Physical Therapy in Sport, v. 13, n. 3, p. 163-169, 2012. ISSN 1466-853X. Disponível em: $<$ http://www.sciencedirect.com/science/article/pii/ S1466853X11000691>.

COJOCARIU, A. Measurement of reaction time in qwan ki do. Biology of Sport, v. 28, n. 2, p. 139-143, 2011. ISSN 0860-021X. Disponível em: $<$ Go to ISI $>$ :// WOS:000293048800011>.

DANE, S.; HAZAR, F.; TAN, U. Correlations between eye-hand reaction time and power of various muscles in badminton players. International Journal of Neuroscience, v. 118, n. 3, p. 349-354, Mar 2008. ISSN 0020-7454. Disponível em: $<<$ Go to ISI $>$ :// WOS:000253720600004 >.

DIETRICH, A.; AUDIFFREN, M. The reticularactivating hypofrontality (RAH) model of acute exercise. Neuroscience and Biobehavioral Reviews, v. 35, n. 6, p. 1305-1325, May 2011. ISSN 0149-7634. Disponível em: < $<$ Go to ISI $>$ ://WOS:000290976600001 >.

DRAPER, S.; MCMORRIS, T.; PARKER, J. K. Effect of acute exercise of differing intensities on simple and choice reaction and movement times. Psychology of Sport and Exercise, v. 11, n. 6, p. 536-541, Nov 2010.
ISSN 1469-0292. Disponível em: $<<$ Go to ISI $>$ :// WOS:000283108700017>.

ECKNER, J. T.; KUTCHER, J. S.; RICHARDSON, J. K. Between-Seasons Test-Retest Reliability of Clinically Measured Reaction Time in National Collegiate Athletic Association Division I Athletes. Journal of Athletic Training, v. 46, n. 4, p. 409-414, Jul-Aug 2011. ISSN 1062-6050. Disponível em: $<<$ Go to ISI $>$ :// WOS:000293983200011>.

ECKNER, J. T. et al. Can a Clinical Test of Reaction Time Predict a Functional Head-Protective Response? Medicine and Science in Sports and Exercise, v. 43, n. 3, p. 382387, Mar 2011. ISSN 0195-9131. Disponível em: $<<$ Go to ISI $>$ ://WOS:000287443200002 >.

ELLEMBERG, D.; ST-LOUIS-DESCHENES, M. The effect of acute physical exercise on cognitive function during development. Psychology of Sport and Exercise, v. 11, n. 2, p. 122-126, Mar 2010. ISSN 1469-0292. Disponível em: $<<$ Go to ISI $>$ ://WOS:000275010500006 >

ERLANGER, D. et al. Development and validation of a web-based neuropsychological test protocol for sportsrelated return-to-play decision-making. Archives of Clinical Neuropsychology, v. 18, n. 3, p. 293-316, Apr 2003. ISSN 0887-6177. Disponível em: $<<$ Go to ISI $>$ :// WOS:000182166200005 >.

GENTIER, I. et al. A comparative study of performance in simple and choice reaction time tasks between obese and healthy-weight children. Research in Developmental Disabilities, v. 34, n. 9, p. 2635-2641, Sep 2013. ISSN 0891-4222. Disponível em: $<<$ Go to ISI $>$ :// WOS:000322423200023>.

GONZALEZ, J. G.; TUBIO, J. C. C.; SANCHEZ, J. C. J. Practice of physical activity and speed of cognitive processing in the elderly. Revista Internacional De Medicina Y Ciencias De La Actividad Fisica Y Del Deporte, v. 11, n. 44, p. 803-816, Dec 2011. ISSN 1577-0354. Disponível em: $<$ Go to ISI $>$ :// WOS:000299728800011>.

GUNENDI, Z.; TASKIRAN, O. O.; BEYAZOVA, M. What is the optimal repetition number in electromyographic reaction time studies? Clinical Biomechanics, v. 20, n. 7, p. 754-758, Aug 2005. ISSN 0268-0033. Disponível em: < $<$ Go to ISI $>$ ://WOS:000230523500013 > .

ISHIHARA, M.; IMANAKA, K.; MORI, S. Lateralized effects of target location on reaction times when preparing for manual aiming at a visual target. Human Movement Science, v. 21, n. 5-6, p. 563-582, 2002. ISSN 0167-9457. Disponível em: < http://www.sciencedirect.com/science/ article/pii/S0167945702001732>.

JIMÉNEZ-PAVÓN, D. et al. Effects of a Running Bout in the Heat on Cognitive Performance. Journal of Exercise Science \&amp; Fitness, v. 9, n. 1, p. 58-64, 2011. ISSN 
1728-869X. Disponível em: < http://www.sciencedirect. com/science/article/pii/S1728869X11600087 >.

KAMINSKI, T. W.; GROFF, R. M.; GLUTTING, J. J. Examining the stability of Automated Neuropsychological Assessment Metric (ANAM) baseline test scores. Journal of Clinical and Experimental Neuropsychology, v. 31, n. 6, p. 689-697, 2009. ISSN 1380-3395. Disponível em: < $<$ Go to ISI $>$ ://WOS:000268883300006 >.

KELLY, D. D.; MURPHY, B. A.; BACKHOUSE, D. P. Use of a mental rotation reaction time paradigm to measure the effects of upper cervical adjustments on cortical processing: A pilot study. Journal of Manipulative and Physiological Therapeutics, v. 23, n. 4, p. 246-251, May 2000. ISSN 0161-4754. Disponível em: $<<$ Go to ISI $>$ :// WOS:000087396800004 >.

KELLY, M. P. et al. Assessment of Acute Concussion in the Combat Environment. Archives of Clinical Neuropsychology, v. 27, n. 4, p. 375-388, Jun 2012. ISSN 0887-6177. Disponível em: $<<$ Go to ISI $>$ :// WOS:000304198300001 >.

KIDA, N.; ODA, S.; MATSUMURA, M. Intensive baseball practice improves the Go/Nogo reaction time, but not the simple reaction time. Cognitive Brain Research, v. 22, n. 2, p. 257-264, 2005. ISSN 0926-6410. Disponível em: < http://www.sciencedirect.com/science/article/pii/ S0926641004002459>.

KORNSPAN, A. S. E.W. Scripture and the Yale psychology laboratory: Studies related to athletes and physical activity. Sport Psychologist, v. 21, n. 2, p. 152-169, Jun 2007. ISSN 0888-4781. Disponível em: $<<$ Go to ISI $>$ :// WOS:000247452000002 >.

KVELDE, T. et al. Physiological and Cognitive Mediators for the Association Between Self-reported Depressed Mood and Impaired Choice Stepping Reaction Time in Older People. Journals of Gerontology Series a-Biological Sciences and Medical Sciences, v. 65, n. 5, p. 538-544, May 2010. ISSN 1079-5006. Disponível em: $<<$ Go to ISI>://WOS:000277120700012>.

LO BUE-ESTES, C. et al. Short-term exercise to exhaustion and its effects on cognitive function in young women. Perceptual and Motor Skills, v. 107, n. 3, p. 933-945, Dec 2008. ISSN 0031-5125. Disponível em: <Go to ISI $>$ :// WOS:000262691000027>.

LORD, S. R. et al. The effect of group exercise on physical functioning and falls in frail older people living in retirement villages: A randomized, controlled trial. Journal of the American Geriatrics Society, v. 51, n. 12, p. 16851692, Dec 2003. ISSN 0002-8614. Disponível em: $<<$ Go to ISI $>$ ://WOS:000186562400001 >.

LOUREIRO, L. D. B.; DE FREITAS, P. B. Influence of the performance level in badminton players in neuromotor aspects during a target-pointing task. Revista Brasileira
De Medicina Do Esporte, v. 18, n. 3, p. 203-207, May-Jun 2012. ISSN 1517-8692. Disponível em: $<<$ Go to ISI $>$ :// WOS:000308370500014 >.

LUFT, C. D.; TAKAS, E.; DARBY, D. Heart rate variability and cognitive function: Effects of physical effort. Biological Psychology, v. 82, n. 2, p. 196-201, Oct 2009. ISSN 0301-0511. Disponível em: < < Go to ISI $>$ :// WOS:000270380200012>.

MCDONNELL, M. N.; SMITH, A. E.; MACKINTOSH, S. F. Aerobic Exercise to Improve Cognitive Function in Adults With Neurological Disorders: A Systematic Review. Archives of Physical Medicine and Rehabilitation, v. 92, n. 7, p. 1044-1052, Jul 2011. ISSN 0003-9993. Disponível em: $<<$ Go to ISI $>$ ://WOS:000292615900004 >.

MOHER, D. et al. Preferred reporting items for systematic reviews and meta-analyses: the PRISMA statement. British Medical Journal, v. 339, Jul 21 2009. ISSN 0959-535X. Disponível em: $<<$ Go to ISI $>$ ://WOS:000268351400018 $>$.

MURRAY, N. P.; RUSSONIELLO, C. Acute Physical Activity on Cognitive Function: A Heart Rate Variability Examination. Applied Psychophysiology and Biofeedback, v. 37, n. 4, p. 219-227, Dec 2012. ISSN 1090-0586. Disponível em: $<<$ Go to ISI $>$ :// WOS:000310536800001 >

NEWSON, R. S.; KEMPS, E. B. Relationship between fitness and cognitive performance in younger and older adults. Psychology \& Health, v. 23, n. 3, p. 369-386, 2008. ISSN 0887-0446. Disponível em: $<<$ Go to ISI $>$ :// WOS:000253508000007>.

O'BRIEN, D. E. The relation between reaction time and the type and complexity of the succeeding task. 1959.

OKAGAWA, S.; KUBOTA, K. Jogging improved performance of a behavioral branching task: implications for prefrontal activation. Neuroscience Research, v. 49, n. 3, p. 325-337, Jul 2004. ISSN 0168-0102. Disponível em: < $<$ Go to ISI $>$ ://WOS:000222516100007 >.

REGISTER-MIHALIK, J. et al. The effects of headache on clinical measures of neurocognitive function. Clinical Journal of Sport Medicine, v. 17, n. 4, p. 282-288, Jul 2007. ISSN 1050-642X. Disponível em: $<<$ Go to ISI $>$ :// WOS:000247948500009>.

REUTERS, T. Web of Knowledge - About - What It Is. 2013. Disponível em: < http://wokinfo.com/about/ whatitis/\#>. Acesso em: jan.

SCHMIDT, J. D. et al. Identifying Impairments after Concussion: Normative Data versus Individualized Baselines. Medicine and Science in Sports and Exercise, v. 44, n. 9, p. 1621-1628, Sep 2012. ISSN 0195-9131. Disponível em: $<<$ Go to ISI $>$ ://WOS:000307624600001 $>$.

SHIPLEY, B. A. et al. Association between mortality and 
cognitive change over 7 years in a large representative sample of UK residents. Psychosomatic Medicine, v. 69, n. 7, p. 640-650, Sep 2007. ISSN 0033-3174. Disponível em:

$<<$ Go to ISI $>$ ://WOS:000249881000008 $>$.

SKURVYDAS, A. et al. Muscle Damaging Exercise Affects Isometric Force Fluctuation as well as Intraindividual Variability of Cognitive Function. Journal of Motor Behavior, v. 42, n. 3, p. 179-186, May-Jun 2010. ISSN 0022-2895. Disponível em: $<<$ Go to ISI $>$ :// WOS:000278651000004 >.

Relationship between simple reaction time and body mass index. Homo-Journal of Comparative Human Biology, v. 60, n. 1, p. 77-85, 2009. ISSN 0018-442X.

Disponível em: $<<$ Go to ISI $>$ :/WOS:000263268100005 >.

SMILEY-OYEN, A. L. et al. Exercise, Fitness, and Neurocognitive Function in Older Adults: The "Selective Improvement" and "Cardiovascular Fitness" Hypotheses. Annals of Behavioral Medicine, v. 36, n. 3, p. 280-291, Dec 2008. ISSN 0883-6612. Disponível em: $<<$ Go to ISI $>$ ://WOS:000261786100008 >

VANTTINEN, T. et al. Effects of age and soccer expertise on general tests of perceptual and motor performance among adolescent soccer players. Perceptual and Motor Skills, v. 110, n. 3, p. 675-692, Jun 2010. ISSN 0031-5125. Disponível em: <<Go to ISI >:/WOS:000279282400001 >.

YOTANI, K. et al. Response Training Shortens VisuoMotor Related Time in Athletes. International Journal of Sports Medicine, v. 32, n. 8, p. 586-590, Aug 2011. ISSN 0172-4622. Disponível em: $<<$ Go to ISI $>$ :// WOS:000293460100005 > 